\title{
Efficacy of Oral Cryotherapy During Oxaliplatin Infusion in Preventing Oral Thermal Hyperalgesia: A Randomized Trial
}

\author{
Brittany Bauman, BA'; Rosemarie Mick, MSª,b; Eileen Martinez, BSN, RN, OCNa; Theresa M. Lawless, BSN, RN, OCNª \\ Lindsey Zinck, MSN, RN, OCNa; Paige Sinclair, MSN, RN, OCNa; Mary Fuhrer, BA; ; Mark O'Hara, MD ${ }^{a, c}$;

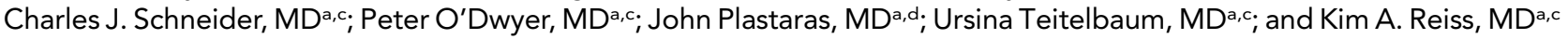

\section{ABSTRACT}

Background: Chemotherapy-induced oral thermal hyperalgesia (OTH) is a common and debilitating side effect of platinum-based anticancer agents. This study evaluated the efficacy of oral cryotherapy in preventing OTH during oxaliplatin chemotherapy infusion. Methods: Patients with gastrointestinal cancer treated with biweekly oxaliplatin ( $85 \mathrm{mg} / \mathrm{m}^{2}$ over 120 minutes) at Abramson Cancer Center at the University of Pennsylvania were randomized to receive oral cryotherapy (ice chips) during oxaliplatin infusion or standard-of-care treatment. All patients completed baseline questionnaires regarding oral and peripheral symptoms and on-treatment questionnaires on day 1 of each subsequent chemotherapy cycle. Those in the treatment arm were asked to document how long they kept the ice chips in their mouths $(0,<30,30,60,90$, or 120 minutes $)$ and to report their discomfort associated with oral cryotherapy. Evaluable patients were those who had completed at least 2 cycles of oxaliplatin therapy. Results: Of 62 randomized patients with a variety of gastrointestinal malignancies, 50 (25 per treatment arm) were evaluable for efficacy. The rate of patients with oral symptoms after the first treatment cycle was significantly lower in the intervention arm $(n=8 ; 32 \%)$ than in the control arm $(n=18 ; 72 \%)$, meeting the primary study objective $(P=.01)$. The magnitude of difference in symptom scores before versus after the first treatment cycle was significantly less in the intervention versus control arm $(P=.001)$. No difference in oral symptoms over time was seen between the intervention and control groups $(P=.20)$, although a high attrition rate was noted. Duration of ice chip exposure was associated with improved oral symptoms over time $(P=.02)$. Conclusions: Oral cryotherapy is a tolerable and cost-effective method of diminishing OTH in patients receiving oxaliplatin chemotherapy, and seems to be most effective in the early stages of treatment.

J Natl Compr Canc Netw 2019;17(4):358-364 doi: 10.6004/jnccn.2018.7110

${ }^{a}$ Abramson Cancer Center, ${ }^{b}$ Department of Biostatistics, Epidemiology, and Informatics, 'Department of Medicine, and ${ }^{\mathrm{d} D e p a r t m e n t}$ of Radiation Oncology, Perelman School of Medicine, University of Pennsylvania, Philadelphia, Pennsylvania.

\section{Background}

Oxaliplatin is a commonly used chemotherapy for patients with gastrointestinal cancers. ${ }^{1,2} \mathrm{~A}$ unique and common toxicity of oxaliplatin is oral thermal hyperalgesia $(\mathrm{OTH})$, a form of acute neuropathy caused by disruption of voltage-gated $\mathrm{Na}^{+}$channels that leads to hyperexcitability of cold-sensing sensory nerves. ${ }^{3-5} \mathrm{OTH}$ is known to reduce quality of life, and no established strategies exist to prevent this toxicity. ${ }^{4}$ Recently, it has been shown that cooling of anatomic areas during chemotherapy infusion can reduce toxicity to those regions, presumably because of local vasoconstriction. A randomized trial by Nangia et $\mathrm{al}^{6}$ showed that the application of a "cold cap" during chemotherapy infusion reduced alopecia up to $50 \%$ in patients with breast cancer. In another trial by Hanai et $\mathrm{al}^{7}{ }^{7}$ the application of a limb-cooling device to one hand during paclitaxel infusion reduced the development of peripheral neuropathy (PN) compared with the untreated hand. In addition, several clinical trials have shown that oral cryotherapy reduces mucositis in patients with cancer receiving 5-fluorouracil-based chemotherapy or high-dose melphalan before hematopoietic stem cell transplant. ${ }^{8-10} \mathrm{We}$ therefore hypothesized that oral cryotherapy delivered during oxaliplatin infusion would reduce the intensity and duration of OTH by limiting chemotherapy delivery to the oral mucosa.

\section{Methods}

\section{Study Design}

A single-institution, randomized, controlled trial was conducted to evaluate the efficacy of oral cryotherapy during oxaliplatin infusion as a preventive measure against OTH. Patients with gastrointestinal malignancies who were scheduled to receive full-dose oxaliplatincontaining chemotherapy for a minimum of 4 planned

See JNCCN.org for supplemental online content. 
cycles were assessed for eligibility. Patients who had received prior platinum therapy, had dentures, or had a chronic condition of the oral cavity, such as active herpes simplex infection or chronic dry mouth, were excluded. After informed consent was obtained and before oxaliplatin was started, patients were enrolled and randomized in a 1:1 allocation to either cryotherapy or control. On each treatment day, those randomized to the intervention arm were provided with ice chips continuously during each 120-minute oxaliplatin infusion and were asked to keep ice in their mouths as consistently as possible during this time. Subjects in the control arm were asked to avoid ingesting anything cooler than room temperature during oxaliplatin infusion. Baseline demographics and treatment histories were collected via review of the electronic medical record. Surveys derived from the validated Leeds Assessment of Neuropathic Symptoms and Signs (LANSS) pain scale ${ }^{11}$ addressing oral and peripheral symptoms were completed by all patients at baseline (cycle 1, day 1 before chemotherapy) and at every subsequent chemotherapy infusion (supplemental eAppendices 1 and 2, available with this article at JNCCN.org). Symptoms were scored using a 5-point Likert scale $(1=$ no symptoms; $5=$ maximal symptoms $)$. Oral symptoms were defined as oral sensations of prickling, burning, tingling, or pins and needles, either at baseline or induced by contact with cold foods or beverages. Peripheral symptoms were defined as sensations of prickling, burning, tingling, or pins and needles in the hands and feet.

Patients in the intervention arm were asked to log the length of time they were able to keep ice in their mouths $(0,<30,30,60,90$, or 120 minutes) and to rate their level of discomfort during this activity (not uncomfortable, mildly uncomfortable, somewhat uncomfortable, very uncomfortable, or severely uncomfortable). Provider documentation regarding any clinical evidence of mucositis or PN was recorded. Changes in oxaliplatin dosing or schedule and reasons for the changes were recorded. This trial was conducted at Abramson Cancer Center at the University of Pennsylvania and approved by the local Institutional Review Board.

\section{Statistical Considerations}

The primary end point was oral symptoms at cycle 2 (ie, after the first treatment cycle), because patients did not have oral symptoms at baseline before starting oxaliplatin. For each individual symptom question, scores ranged from 1 (no symptom) to 5 (maximal symptom). Presence of oral symptoms was scored as the summation of 6 individual oral symptom questions. Thus, a sum score of 6 indicated that the patient did not have oral symptoms after the first treatment cycle, whereas a sum score $>6$ reflected some level of oral symptoms. With
25 patients in each treatment arm, this study was designed to have $80 \%$ power to detect a decrease in the rate of patients with oral symptoms after the first treatment cycle from $80 \%$ in the control arm to $40 \%$ in the intervention arm by Fisher exact test using a 2-sided $5 \%$ type I error rate.

Baseline characteristics were summarized for each treatment arm. Continuous variables were summarized by mean, standard error, and range. Categorical variables were summarized by frequency and percentage. Continuous variables were compared between arms using Student $t$ test, and categorical variables were compared using Fisher exact test.

At baseline, the symptom survey had 6 oral and 6 peripheral symptom questions (eAppendix 1). With a symptom score of 1 to 5 for each of the individual symptom questions, the possible range for the symptom sum score at baseline was 6 to 30; sum scores were computed separately for oral and peripheral symptoms. For treatment cycles 1 to 12 , the symptom survey had 7 oral and 6 peripheral symptom questions (eAppendix 2). Possible ranges for the symptom sum score were 7 to 35 for oral symptoms and 6 to 30 for peripheral symptoms. To compare baseline with cycle 2 (ie, after the first treatment cycle), the oral sum score was derived on the same 6 oral symptom questions asked at baseline. The difference in symptom sum scores between baseline and cycle 2 was calculated and compared between treatment arms using the Student $t$ test. The difference in symptom scores between baseline and cycle 2 was also calculated for each individual symptom. These differences ranged from -3 (ie, better symptoms at cycle 2) to 4 (ie, worse symptoms at cycle 2). Differences were compared between treatment arms using Wilcoxon rank-sum test. To model trends over time in symptoms reported at treatment cycles 1 to 12 , the oral sum score was the summation of all 7 oral symptom questions. To account for within-subject correlation in longitudinal symptom scores, a linear mixed effects model was used to examine trends over time in oral and peripheral symptom sum scores using the mixed command in STATA, version 15 (StataCorp LP). The impact of treatment arm (ie, time-by-treatment interaction) was tested using a likelihood ratio test, and the impact of ice duration (a 6-level ordinal covariate) on oral and peripheral symptom sum scores over time was assessed using a linear mixed effects model based on data from the intervention arm.

All $P$ values were 2 -sided, and $P<.05$ was considered statistically significant. No adjustment was made for multiple testing of the individual oral and peripheral symptom scores. All analyses were performed using either SPSS Statistics, version 25 (IBM Corporation) or STATA, version 15. 


\section{Results}

\section{Patient Recruitment and Characteristics}

A total of 65 patients were assessed for eligibility. Two patients refused to participate and a third was ineligible. Of the 62 patients randomized, 12 were nonevaluable because of discontinuation of platinum therapy before cycle $2(n=2)$, death $(n=2)$, withdrawal of consent due to intolerance of cryotherapy $(n=1)$, or withdrawal of consent for other reasons $(n=7)$. The remaining 50 patients were included in the final analysis (Figure 1). Between cycles 2 and 12 of therapy, patient attrition occurred due to various chemotherapy- or cancerrelated reasons (supplemental eTable 1).

Baseline demographics of the evaluable patients are displayed in Table 1. Patients in the intervention arm had a mean age of 60.2 years (range, 33-76 years) and were predominantly men $(56 \%)$. Most had a diagnosis of colorectal cancer (52\%), followed by pancreatic $(32 \%)$, gastric $(8 \%)$, small bowel $(4 \%)$, and other cancers $(4 \%)$. Patients in the control arm had a mean age of 58.40 years (range, 35-79 years) and were predominantly men (64\%). The most common malignancy was colorectal cancer (44\%), followed by pancreatic $(20 \%)$, other $(20 \%)$, esophageal $(8 \%)$, and gastric cancers $(8 \%)$. In the intervention group, $40 \%$ of patients were receiving chemotherapy in the palliative setting, and $60 \%$ were receiving therapy in the neoadjuvant or adjuvant setting. In the control group, $60 \%$ were receiving treatment in the palliative setting, and $40 \%$ were receiving therapy in the neoadjuvant or adjuvant setting. In the intervention arm, $28 \%$ had received prior chemotherapy versus $12 \%$ in the control group. Most patients in both groups ( $76 \%$ and $72 \%$, respectively) did not carry a diagnosis of diabetes mellitus. Oxaliplatin was reduced in 2 of the 50 patients (both in the intervention arm) and therefore was not considered a confounding factor.

\section{Oral Cold Sensitivity}

At baseline, none of the patients in either treatment arm reported any ongoing oral symptoms. Thus, sum scores were 6.0 for all patients (Table 1). The rate of patients with oral symptoms (sum score $>6.0$ ) after the first treatment cycle was significantly lower in the intervention arm $(n=8 ; 32 \%)$ than in the control arm $(\mathrm{n}=18 ; 72 \%)$, meeting the primary study objective (Fisher exact test, $P=.01$ ). When examining the magnitude of difference between baseline and cycle 2 for oral symptom sum scores, we observed that patients in the intervention group had statistically significantly fewer oral symptoms than those in the control group (mean \pm SE, $1.12 \pm 0.38$ vs $4.80 \pm 0.88 ; P=.001$ ) (Table 2). Although both groups reported the presence of oral sensations (oral question $1 ; P=.127$ ), the intervention group reported statistically significantly less exacerbation of symptoms with oral cold exposure (oral question 2; $P=.001$ ), less difficulty in eating and drinking cold items (oral question $3 ; P<.001$ ), less difficulty eating and drinking overall (oral question 4;

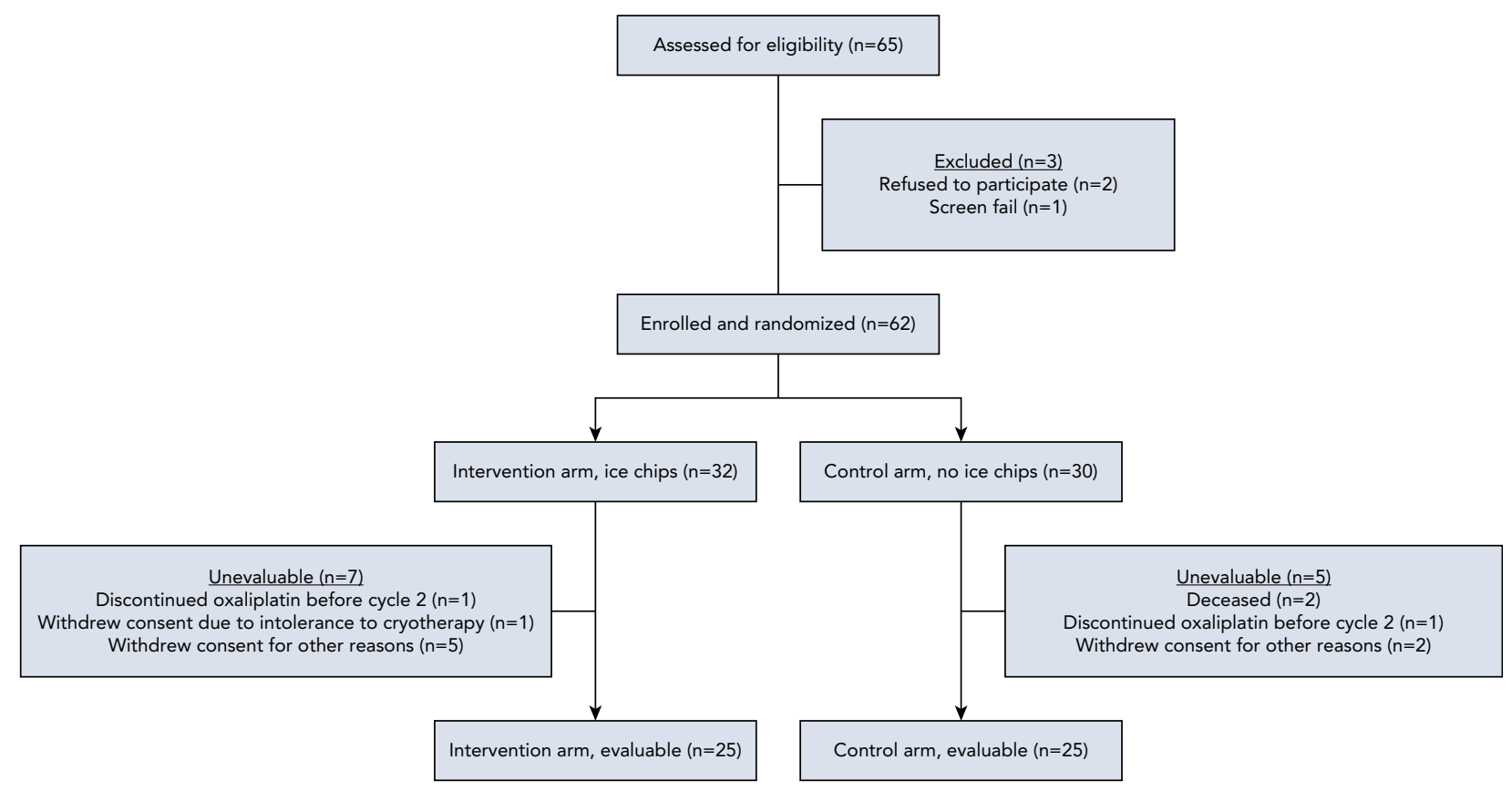

Figure 1. CONSORT diagram. 


\section{Table 1. Baseline Patient Characteristics and Symptom Sum Scores}

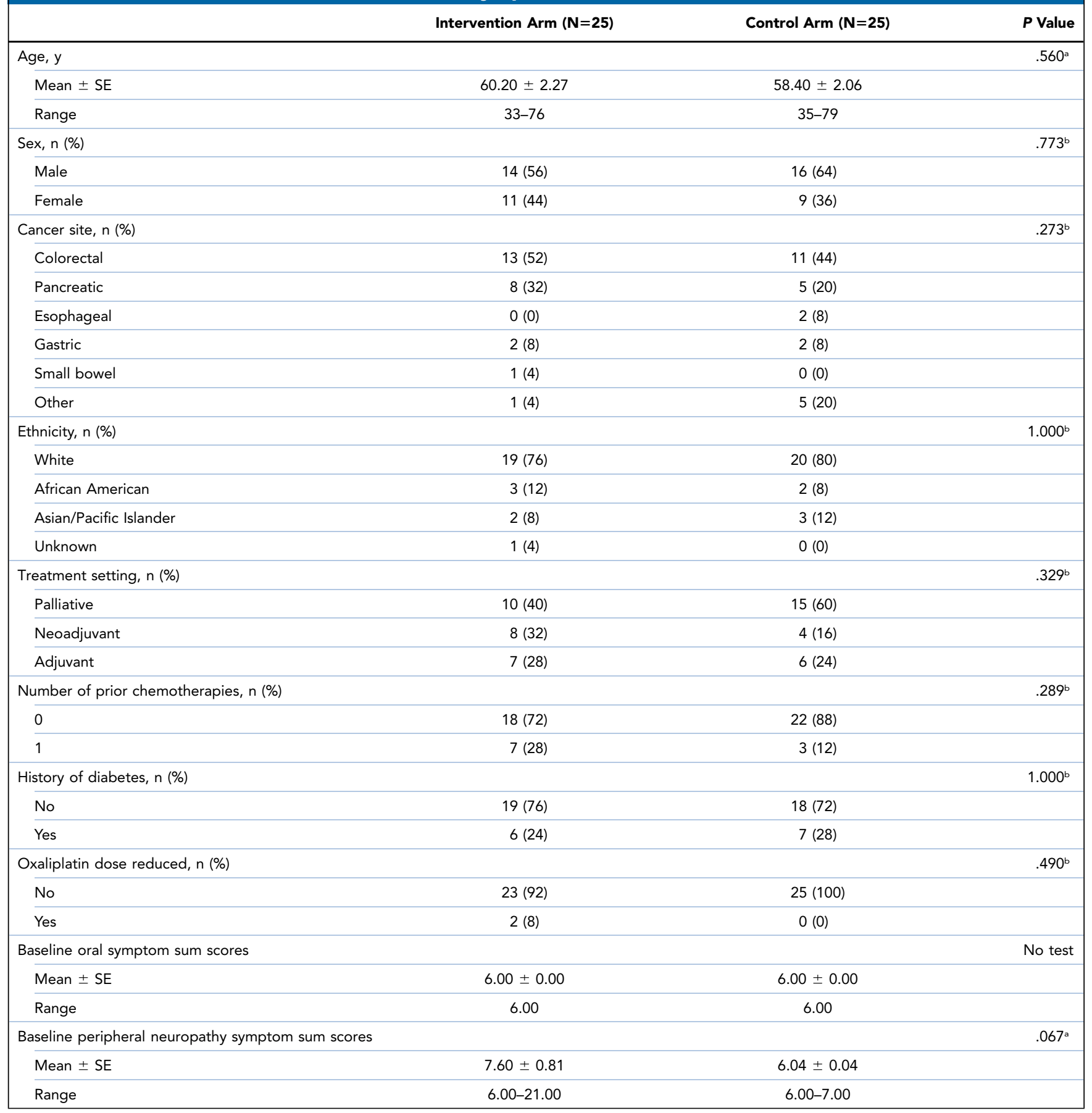

aStudent $t$ test $P$ value.

${ }^{\mathrm{b}}$ Fisher exact test $P$ value.

$P=.001)$, less negative effect on quality of life due to oral symptoms (oral question $5 ; P<.001$ ), and shorter duration of oral symptoms (oral question $6 ; P=.006$ ) (supplemental eTable 2).

Sum scores of oral symptoms over time were compared between treatment arms. Although oral symptom scores seemed to increase over time in the control arm and plateau in the intervention arm, no significant difference was detected $(P=.20)$ (Figure 2A). Power to detect longitudinal differences was likely reduced because of high attrition in later cycles. 


\begin{tabular}{|c|c|c|c|}
\hline & \multicolumn{3}{|c|}{$\begin{array}{c}\text { Difference in Scores } \\
\text { (Cycle } 2 \text { Minus Baseline) }\end{array}$} \\
\hline & $\begin{array}{l}\text { Intervention Arm } \\
\quad(\mathbf{N}=\mathbf{2 5})\end{array}$ & $\begin{array}{l}\text { Control Arm } \\
(\mathbf{N}=\mathbf{2 5})\end{array}$ & $\begin{array}{c}P \\
\text { Value }\end{array}$ \\
\hline Oral symptom sum scores & & & .001 \\
\hline Mean \pm SE & $1.12 \pm 0.38$ & $4.80 \pm 0.88$ & \\
\hline Range & $0.00-7.00$ & $0.00-14.00$ & \\
\hline \multicolumn{3}{|c|}{ Peripheral neuropathy symptom sum scores } & .001 \\
\hline Mean \pm SE & $0.04 \pm 0.65$ & $3.28 \pm 0.66$ & \\
\hline Range & -9.00 to 8.00 & $0.00-10.00$ & \\
\hline
\end{tabular}

aStudent $t$ test.

\section{Peripheral Neuropathy}

At baseline, no statistically significant difference in PN symptoms was seen between the arms, although a trend toward more severe baseline peripheral symptoms was observed in the intervention arm compared with the control arm (mean \pm SE, $7.60 \pm 0.81$ vs $6.04 \pm$ $0.04 ; P=.067$ ) (Table 1). When the difference in peripheral symptom sum scores between baseline and cycle 2 was compared in the treatment arms, we observed that patients in the intervention group had statistically significantly fewer peripheral symptoms than those in the control group (mean \pm SE, $0.04 \pm$ 0.65 vs $3.28 \pm 0.66 ; P=.001$ ) (Table 2 ). All 5 patients in the intervention arm who had peripheral symptoms at baseline had improvement at cycle 2 , with cycle 2 decreases in sum scores of 1 to 9 points. Patients in the intervention arm reported fewer sensations of prickling, burning, or pins and needles (PN question

A

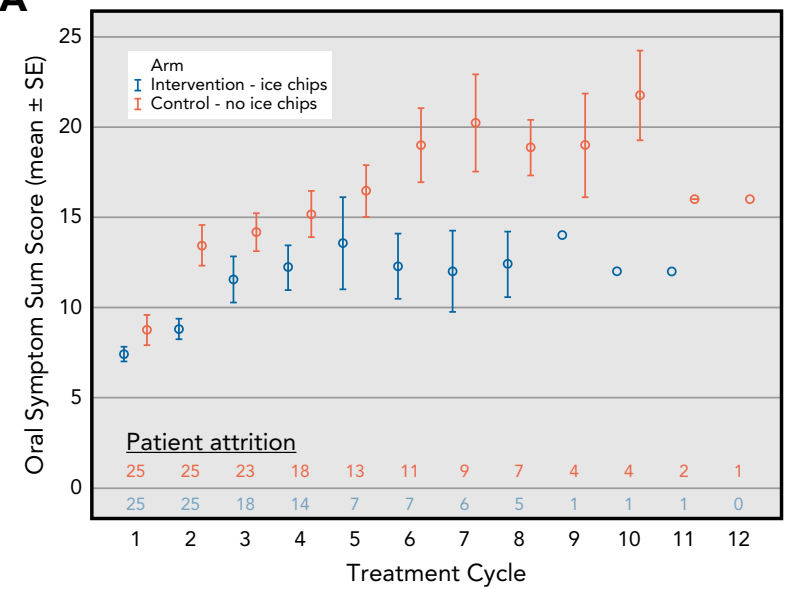

$1 ; P=.006$ ), less sensitivity to touch (PN question 2; $P=.008$ ), less impact of symptoms on work or recreational activities (PN question $3 ; P=.006$ ), less impact on quality of life (PN question $4 ; P=.044$ ), shorter duration of symptoms (PN question 5; $P=.001$ ), and fewer worsening symptoms overall ( $\mathrm{PN}$ question 6: $P=.012$ ) (supplemental eTable 2). Sum scores of peripheral symptoms over time were compared between treatment arms, and no difference was detected $(P=.90)$ (Figure 2B).

\section{Effect of Ice Duration on Oral Symptoms}

Patients in the intervention arm were asked to record the length of ice chip exposure at each cycle via a provided time chart. Length of exposure was categorized as $0,<30,30,60,90$, or 120 minutes. At completion of the first infusion, $12(48 \%), 1$ (4\%), and 5 patients $(20 \%)$ kept the ice chips in their mouths for 120,90 , and 60 minutes, respectively. At completion of the second cycle, fewer patients had completed 120 minutes of ice exposure; 8 (32\%), 1 (4\%), and 9 patients (36\%) kept the ice chips in their mouths for 120,90 , and 60 minutes, respectively. Longer ice duration at each cycle was associated with a reduction in oral symptom sum score over time $(P=.02)$ (Figure 3$)$.

\section{Discussion}

We hypothesized that using oral cryotherapy during infusion with oxaliplatin might limit $\mathrm{OTH}$, a common and troublesome toxicity of this agent. To that end, we randomly assigned patients in a 1:1 fashion to receive either oral cryotherapy (ice chips) during their 120-minute oxaliplatin infusion or no cryotherapy. Patients were
B

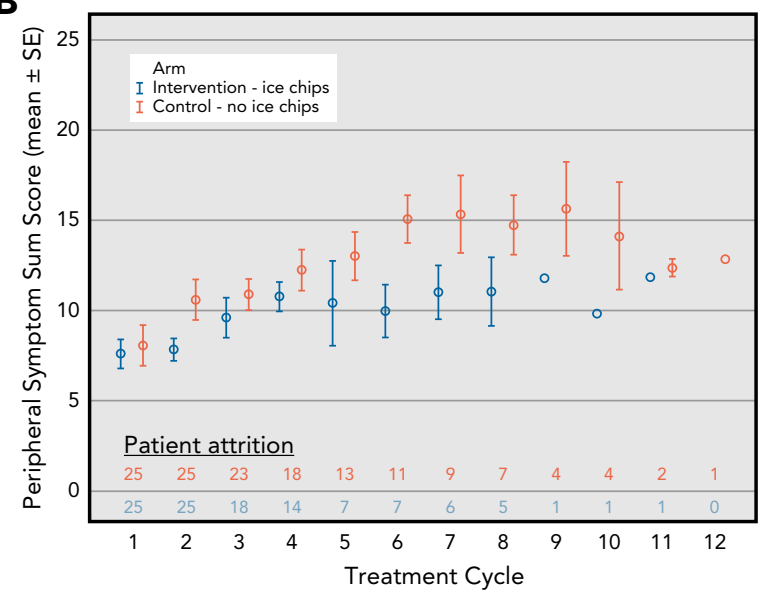

Figure 2. (A) Longitudinal oral symptom sum scores by treatment arm. (B) Longitudinal peripheral symptom sum scores by treatment arm. No difference in oral symptoms $(P=.20)$ or peripheral symptoms $(P=.90)$ is seen between the arms over time, based on likelihood ratio test $P$ value derived from linear mixed effects model. 


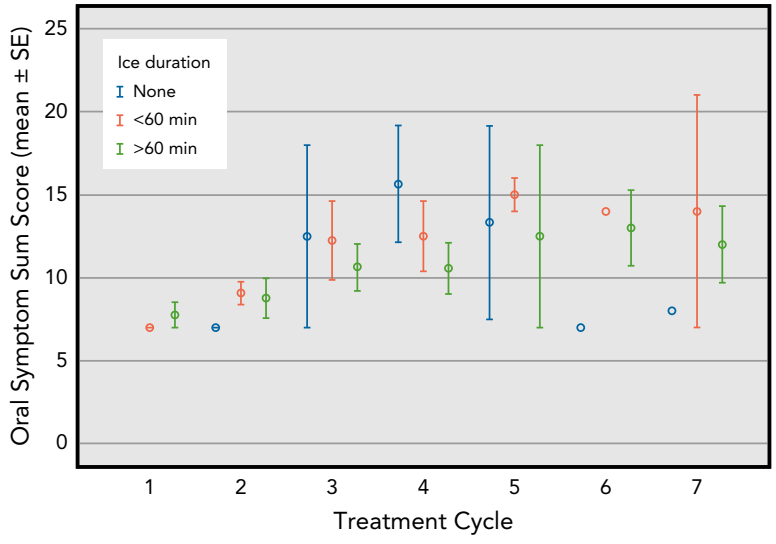

Figure 3. Longitudinal oral symptom sum scores by ice duration for the intervention arm. The mean values of ice duration for each treatment cycle are on or to the right of the dotted line (only the first 7 cycles are shown). Note that in mixed effects regression, the 6 ice duration categories $(0,<30,30,60,90$, and 120 minutes) were modeled individually.

assessed for oral and PN symptoms via serial surveys at baseline and each subsequent chemotherapy cycle, and those in the intervention arm were asked to record how long they used cryotherapy and to rate their level of discomfort during use (supplemental eAppendices 1 and 2). Our findings reveal that between baseline and cycle 2 of oxaliplatin chemotherapy, patients who received oral cryotherapy had a statistically significant reduction in $\mathrm{OTH}$, including severity and duration of symptoms, although this effect seemed to diminish over time.

Patients who completed at least 2 chemotherapy cycles containing oxaliplatin were considered evaluable for efficacy, and participants were followed for a maximum of 12 cycles. Over time, there was substantial attrition as patients discontinued oxaliplatin for a variety of reasons, including chemotherapy toxicity, completion of planned chemotherapy, or disease progression. Therefore, the ability to make comparisons between the intervention and control groups longitudinally was limited by increasingly smaller numbers of patients. Although there may have been continued benefit over time for the individual patients who remained on therapy, we did not observe a statistically significant difference in oral symptoms between arms after cycle 2.
Interestingly, we also observed a self-reported significant reduction in $\mathrm{PN}$ in the intervention arm between baseline and cycle 2 of treatment. A possible and intriguing explanation for this finding relates to a recent study by Onitsuka et al, ${ }^{12}$ in which 8 healthy individuals ingested either a thermoneutral drink or ice slurry for 5 minutes while undergoing rectal, skin, and brain temperature measurements, with the latter measured via proton MR spectroscopy. After only 5 minutes of oral ice exposure, brain and rectal temperatures both decreased significantly. ${ }^{12}$ It seems reasonable to hypothesize, therefore, that oral cooling with ice exposure might lower the core temperature and result in secondary peripheral vasoconstriction. If this were the case, chemotherapyinduced PN could theoretically be reduced by oral exposure to ice during treatment. Alternatively, the corelation of OTH and PN may be a result of a lowered nociceptive threshold due to hypersensitivity. ${ }^{13}$

We must also consider that patients in our intervention arm may have experienced at least a degree of placebo effect and that this may have affected reporting of both oral and peripheral symptoms. In this particular setting, conducting a blinded study was not feasible. To further address the issue of possible placebo effect, future studies should be designed to include objective markers, such as temperature changes of the core, skin, and oral mucosa of patients in both groups. Comparing objective changes in temperatures with subjective symptom reduction reporting could further clarify this issue.

\section{Conclusions}

Results of our study show that oral cryotherapy during oxaliplatin infusion has the potential to reduce OTH and PN symptoms caused by this chemotherapy, at least during early cycles of treatment. Reduction in OTH has the potential to improve the quality of life in patients undergoing oxaliplatin treatment.

Submitted August 29, 2018; accepted for publication November 21, 2018.

Disclosures: The authors have not received any financial consideration from any person or organization to support the preparation, analysis, results, or discussion of this article.

Funding: This work was supported by the Penn Innovation Project (B.B., K.A.R.) and Abramson Cancer Center Support Grant P30-CA016520 (R.M.).

Correspondence: Kim A. Reiss, MD, Perelman Center for Advanced Medicine University of Pennsylvania Perelman School of Medicine, 3400 Civic Center Boulevard, Philadelphia, PA 19104. Email: kim.reissbinder@uphs.upenn.edu

\section{References}

1. André $T$, de Gramont A, Vernerey D, et al. Adjuvant fluorouracil, leucovorin, and oxaliplatin in stage II to III colon cancer: updated 10-year survival and outcomes according to BRAF mutation and mismatch repair status of the MOSAIC study. J Clin Oncol 2015;33:4176-4187.

2. Conroy $T$, Desseigne $F$, Ychou $M$, et al. FOLFIRINOX versus gemcitabine for metastatic pancreatic cancer. N Engl J Med 2011;364:1817-1825.

3. Adelsberger H, Quasthoff S, Grosskreutz J, et al. The chemotherapeutic oxaliplatin alters voltage-gated $\mathrm{Na}^{+}$channel kinetics on rat sensory neurons. Eur J Pharmacol 2000;406:25-32.

4. Descoeur J, Pereira V, Pizzoccaro A, et al. Oxaliplatin-induced cold hypersensitivity is due to remodelling of ion channel expression in nociceptors. EMBO Mol Med 2011;3:266-278. 
5. Attal N, Bouhassira D, Gautron M, et al. Thermal hyperalgesia as a marker of oxaliplatin neurotoxicity: a prospective quantified sensory assessment study. Pain 2009;144:245-252.

6. Nangia J, Wang T, Osborne C, et al. Effect of a scalp cooling device on alopecia in women undergoing chemotherapy for breast cancer: the SCALP randomized clinical trial. JAMA 2017;317:596-605.

7. Hanai $A$, Ishiguro $H$, Sozu $T$, et al. Effects of cryotherapy on objective and subjective symptoms of paclitaxel-induced neuropathy: prospective selfcontrolled trial. J Natl Cancer Inst 2018;110:141-148.

8. Riley P, McCabe MG, Glenny AM. Oral cryotherapy for preventing oral mucositis in patients receiving cancer treatment. JAMA Oncol 2016;2:1365-1366.

9. Peterson DE, Ohrn K, Bowen J, et al. Systematic review of oral cryotherapy for management of oral mucositis caused by cancer therapy. Support Care Cancer 2013;21:327-332.
10. Wang L, Gu Z, Zhai R, et al. Efficacy of oral cryotherapy on oral mucositis prevention in patients with hematological malignancies undergoing hematopoietic stem cell transplantation: a metaanalysis of randomized controlled trials. PLoS One 2015;10: e0128763.

11. Bennett M. The LANSS pain scale: the Leeds Assessment of Neuropathic Symptoms and Signs. Pain 2001;92:147-157.

12. Onitsuka $S$, Nakamura $D$, Onishi $T$, et al. Ice slurry ingestion reduces human brain temperature measured using non-invasive magnetic resonance spectroscopy. Sci Rep 2018;8:2757.

13. Berger JV, Knaepen L, Janssen SP, et al. Cellular and molecular insights into neuropathy-induced pain hypersensitivity for mechanismbased treatment approaches. Brain Res Brain Res Rev 2011;67: 282-310.

\section{See JNCCN.org for supplemental online content.}

